\title{
Multi-Functional Textile Coatings Based on Polymeric Materials
}

\author{
Semih KAZANÇ, Gökhan KAPLAN, Agamirze HATIMBEYLİ, Özgür AKDEMİR \\ Sanko Tekstil İsletmeleri A.Ş. İSKO Şubesi, AR-GE Merkezi, İnegöl, Bursa, Turkey
}

\begin{abstract}
Waterborne-polyurethane dispersions, consisting of polyol and polyisocyanates building blocks are conventional finish materials for various applications in textile industry, particularly in denim industry. This study aims to prepare a multifunctional polyol block, which is compatible with commercially available polyisocyanates, for polyurethane formation. Therefore, well-defined polymers having both hydroxyl and tertiary amine functions prepared via controlled radical polymerization technique namely atom transfer radical polymerization (ATRP). Hydroxyl functions are considered as cross-linking domains with polyisocyanate to form polyurethane and tertiary amine moieties are considered as quaternarization domains with different length of alkyl-bromides. 1H-NMR and Dynamic light scattering (DLS) techniques are used for structural characterization and thermal stability evaluation of synthesized polymers respectively. Those synthesized materials are primarily used for surface modification of cellulosic fabrics to achieve wash-fast antimicrobial coatings. Dyeing studies with indigo dyestuff is conducted with both polymer treated and non-treated fabrics. Dyeing studies showed dye-uptake capabilities of polymer coated samples are found to be higher than non-treated fabrics. Datacolor spectrophotometer SF 600 Plus is used to evaluate color measurements.
\end{abstract}

Keywords: 2-Hydroxyethyl methacrylate (HEMA), 2-(Dimethyl amino) ethyl methacrylate (DMAEMA), ATRP, Antimicrobial, Indigo, LCST.

\section{INTRODUCTION}

Currently, global trends in the textile industry are focused on developing and manufacturing functional textile products with high added-value. The coating of textile substrates with nano- and micro particles constitutes a potential pathway to the production of highly active surfaces for protection against UV radiation, antimicrobial properties, flame retardancy, water repellency and self-cleaning, while preserving the fabrics' appearance and comfort [1]. There are lots of ways to achieve such properties. Living/Controlled free radical polymerization is one of them individually Atom Transfer Radical Polymerization (ATRP) is the most powerful, versatile, simple and inexpensive method in living/controlled radical polymerization [2].

\section{MATERIALS}

2-Hydroxyethyl methacrylate (HEMA) 97\%, 2-(Dimethylamino)ethyl methacrylate (DMAEMA) 98\%, $\mathrm{N}, \mathrm{N}, \mathrm{N}^{\prime}, \mathrm{N}^{\prime \prime}, \mathrm{N}^{\prime \prime}$-Pentamethyldiethylenetriamine (PMDETA) 99\%, -Bromoisobutyryl bromide (BrIBBr) 98\%, n-Hexane
95\%, Ethanol (Technical grade), 1-bromodecane 98\%, all reactants are used as they are received from the supplier.

\section{EXPERIMENTALS}

ATRP is used to prepare random copolymers. HEMA and DMAEMA monomers are polymerized in presence of catalyst system consisting of $\mathrm{Cu}$ (I) $\mathrm{Br}, \mathrm{Cu}$ (II) $\mathrm{Br}$, ligand, and initiator. Polymerizations performed at $60^{\circ} \mathrm{C}$ overnight and stopped through exposing catalyst to air.

Polymerization crude diluted with ethanol and silica gel filtered to remove oxidized $\mathrm{Cu}$ salts. Afterwards, crude precipitated in n-hexane for purification. Due to the fact that reactivity of DMAEMA is higher than HEMA, molar ratio of HEMA is found nearly $\% 3$ in copolymer which is lower than monomer in feed. Quaternizations with different length of alkyl halides were done in dry ethanol to end up antimicrobial copolymers [3, 4]. Compositions of P(DMAEMA-co-HEMA) copolymers were determined with 1H-NMR. Signals belonging to methyl group of DMAEMA and methylene group belonging to HEMA were taken into 
account (Table 1). 1H-NMR (400 MHz, CDCl3): (ppm)= $4.07(2 \mathrm{H},-\mathrm{OCH} 2 \mathrm{CH} 2 \mathrm{~N}(\mathrm{CH} 3) 2,3.82(2 \mathrm{H},-\mathrm{OCH} 2 \mathrm{CH} 2 \mathrm{OH})$, $2.63(2 \mathrm{H},-\mathrm{OCH} 2 \mathrm{CH} 2 \mathrm{OH}), 2.58(2 \mathrm{H},-\mathrm{OCH} 2 \mathrm{CH} 2 \mathrm{~N}(\mathrm{CH} 3) 2$, 2.30 (6H, -N(CH3)2 ), 1.97-1.84 (2H, -RCH2R-), 0.96-0.87 $(3 \mathrm{H},-\mathrm{CCH} 3)$ (Figure 1).

Due to having an optimized number of hydroxyl functions, copolymer \#1 is used for further experiments. First of all, various mass ratios of copolymer 1 and commercially available aliphatic isocyanates ( $\mathrm{NCO} 1$ and $\mathrm{NCO} 2)$ are evaluated with respect to their film formation capability on microscope glass slides (Table 2). Isocyanate NCO1 successfully formed polyurethane films while $\mathrm{NCO} 2$ was failed to form hydrolytically stable films. Polyurethane preparation reactions are completed at $150^{\circ} \mathrm{C}$ for 2 minutes. Due to, insoluble network structure, prepared polyurethane films characterized with FT-IR (Figure 2).

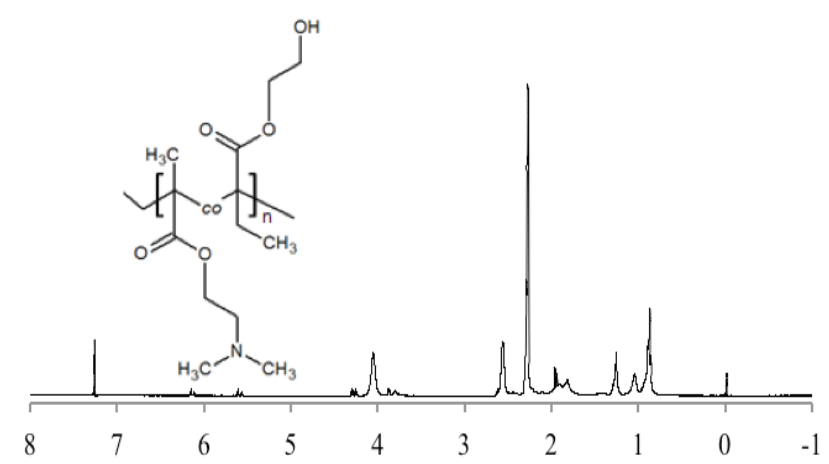

Figure 1. 1H-NMR spectrum of PDMAEMA-co-HEMA copolymer \#1 in Table 1.

Table 1. Composition of synthesized P(DMAEMA-coHEMA) copolymers.

$\begin{array}{ccccccc}\text { \# Copolymer } & \text { F }_{\text {Hema }} & \mathbf{F}_{\text {DMaema }} & \boldsymbol{f}_{\text {Hema }}{ }^{\mathbf{b}} & \boldsymbol{f}_{\text {DMaema }}{ }^{\mathrm{b}} & \text { LCST } \\ \mathbf{1} & 4.76 & 95.24 & 0.09 & 0.91 & 33.80 \\ \mathbf{2} & 88.23 & 11.76 & 0.76 & 0.26 & \text { n.a. } \\ \mathbf{3} & 11.76 & 88.23 & 0.11 & 0.89 & 39.70\end{array}$

a Copolymerization conditions: Copolymer 1: [DMAEMA]/[HEMA]/ $[\mathrm{Cu}(\mathrm{I}) \mathrm{Br}] /[\mathrm{Cu}(\mathrm{II}) \mathrm{Br}] / \mathrm{PMDETA} /[\mathrm{BrIBBr}]=950 / 50 / 20 / 1 / 20 / 5$, Copolymer 2: $[\mathrm{DMAEMA}] /[\mathrm{HEMA}] /[\mathrm{Cu}(\mathrm{I}) \mathrm{Br}] /[\mathrm{Cu}(\mathrm{II}) \mathrm{Br}] / \mathrm{PMDETA} /[\mathrm{BrIBBr}]=$ 120/880/20/1/20/5, Copolymer 3: [DMAEMA]/[HEMA]/[Cu(I)Br]/[Cu(II) $\mathrm{Br}] / \mathrm{PMDETA} /[\mathrm{BrIBBr}]=880 / 120 / 20 / 1 / 20 / 5 .{ }^{\mathrm{d}}$ determined with ${ }^{1} \mathrm{H}-\mathrm{NMR}$.
Table 2. Mass ratios of P(DMAEMA-co-HEMA) copolymer to isocyanate $\mathrm{NCO} 1$ to achieve stable polyurethane film

\begin{tabular}{cccc}
\hline \# Film & $\mathbf{m}_{\text {copolymer 1 }}(\mathbf{m g})$ & $\mathbf{m} \underset{\text { NCO1 }}{(\mathbf{m g})}$ & $\mathbf{\%}$ NCO1 \\
$\mathbf{2}$ & 144.0 & 3.0 & 2.04 \\
$\mathbf{6}$ & 142.0 & 6.0 & 4.05 \\
$\mathbf{4}$ & 144.0 & 16.5 & 10.3 \\
$\mathbf{5}$ & 145.0 & 34.5 & 19.2 \\
\hline
\end{tabular}

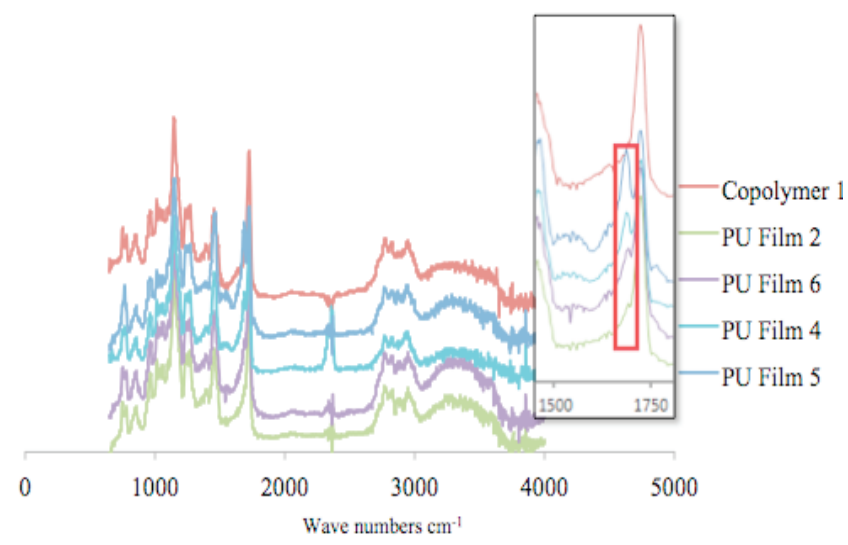

Figure 2. FTIR Spectrums of polyurethane films consisting of P(DMAEMA-co-HEMA) and NCO1.

The peak at $1685 \mathrm{~cm}-1$ corresponds to polyurethane carbonyl (-NHC=OR) bond stretching. PU film 5 containing 19.20 $\% \mathrm{NCO} 1$ has the most intensive PU carbonyl signal, while the PU film 2 containing $2.04 \% \mathrm{NCO} 1$ has the weakest signal at $1685 \mathrm{~cm}-1$ (Figure 2 inset). Peak intensity is found proportional to concentration of NCO1 used to form polyurethane film.

\subsection{Dynamic Light Scattering Measurements}

Temperature trend measurements were performed in between $25^{\circ} \mathrm{C}$ and $45^{\circ} \mathrm{C}$ at various $\mathrm{pH}$ to understand the stability of copolymer and quaternized copolymer solutions with respect to their number size distributions. Solution concentrations for measurements were kept constant $5 \mathrm{~g} / \mathrm{L}$ for all measurements (Figure 3). 


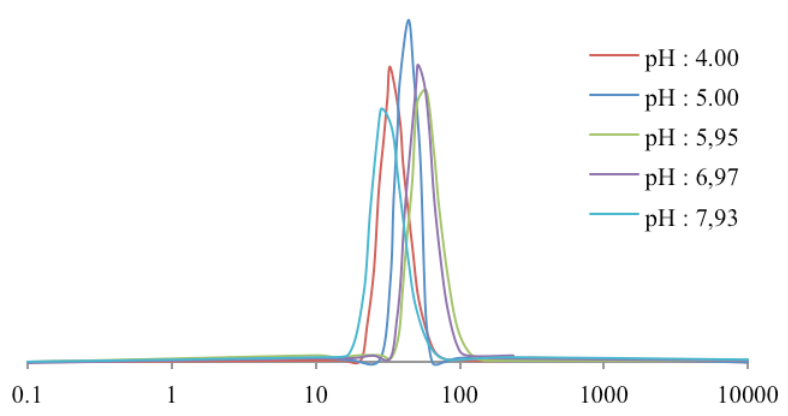

Figure 3. Number distribution of particle sizes belonging to P(DMAEMA-co-HEMA) copolymer at different $\mathrm{pH}$.

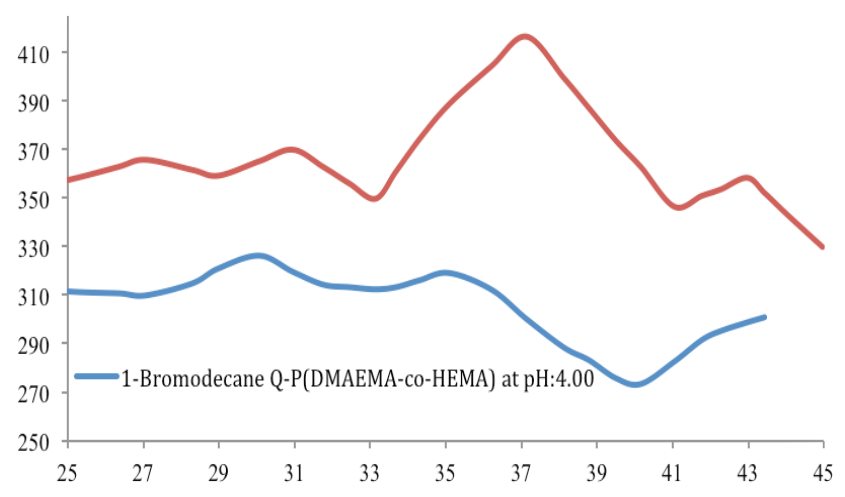

Figure 4. Temperature trend measurement, temperature versus number distribution of particle sizes belonging to 1-bromodecane quaternized P(DMAEMA-co-HEMA) copolymer.

\subsection{Dyeing Studies}

P(DMAEMA-co-HEMA) and 1-bromodecane quaternized P(DMAEMA-co-HEMA) is applied onto non-dyed fabrics as a cationic interface in between fabric surface and indigo dyeing solution. Solution taken from rope dyeing system with a concentration of $1.90 \%$ (owf) is used to dye fabric samples. Due to quaternary amine functionalities fabric surface is positively charged, which attracts negatively charged mono and/or dianionic leuco indigo molecules. In order to, understand the effect of number of dips and color strength 1 passage, 2 passages and 4 passages dyeing studies performed. Dipping and skying times are optimized with respect to oxidation time required for leuco indigo. Kubelka-Munk parameters (K/S) and color strengths are tabulated (Table 3).

\subsection{Color Measurements and Evaluation}

Datacolor SF 600 Plus spectrophotometer operating at $660 \mathrm{~nm}$ is used to evaluate indigo dye uptake capabilities of coated fabrics from indigo dye bath. Lower concentrations of coating formulations containing either P(DMAEMAco-HEMA) copolymer or Q-P(DMAEMA-co-HEMA), is increasing dye uptake (fabric samples 3 and 6 in Table 3 ), on the other hand, increased concentration of both P(DMAEMA-co-HEMA) and Q- P(DMAEMA-co-HEMA) leads to hydrophobicity which repells leuco indigo molecules in dye bath (fabric samples 4 and 7 in Table 3 ).

Table 3. K/S and color strength of indigo dyed, P(DMAEMAco-HEMA) and 1-bromodecane Q-P(DMAEMA-coHEMA) coated fabrics.

\begin{tabular}{|c|c|c|c|c|c|}
\hline & \# Fabric & $\begin{array}{c}C_{\text {polymer }} \\
{[\mathrm{g} / \mathrm{L}]}\end{array}$ & $\begin{array}{c}\text { Color } \\
\text { Strength }\end{array}$ & $\mathbf{K} / \mathbf{S}$ & Samples \\
\hline Non-treated & 1 & 0 & 100 & 8.7 & \\
\hline $\begin{array}{l}\text { P(DMAEMA- } \\
c o \text {-HEMA) }\end{array}$ & 2 & 1 & 116.80 & 10.1 & \\
\hline $\begin{array}{l}\text { P(DMAEMA- } \\
c o \text {-HEMA) }\end{array}$ & 3 & 5 & 122.07 & 10.1 & \\
\hline $\begin{array}{l}\text { P(DMAEMA- } \\
\text { co-HEMA) }\end{array}$ & 4 & 20 & 108.43 & 9.3 & \\
\hline $\begin{array}{l}\text { 1-Brdecane-Q- } \\
\text { P(DMAEMA- } \\
\text { co-HEMA) }\end{array}$ & 5 & 1 & 136.57 & 11.8 & \\
\hline $\begin{array}{l}\text { 1-Brdecane-Q- } \\
\text { P(DMAEMA- } \\
\text { co-HEMA) }\end{array}$ & 6 & 5 & 142.17 & 12.2 & \\
\hline $\begin{array}{l}\text { 1-Brdecane-Q- } \\
\text { P(DMAEMA- } \\
\text { co-HEMA) }\end{array}$ & 7 & 20 & 112.04 & 9.6 & \\
\hline
\end{tabular}

\subsection{Thermo-responsive Coatings}

Synthesized copolymers exhibited an LCST illustrated below (Figure 4). Detailed LCST of polymers studied during the project is listed above (Table 1). Through temperature raise hydrogen bonds formed in between pendant groups 
along the polymer backbone and water molecules are broken (right) and polymer became hydrophobic and opaque. At lowered temperatures where it is sufficient to form hydrogen bonds polymer became hydrophilic and transparent (left). Such a smart behaving material can be used to prepare thermal stimulus responsive textile surfaces.
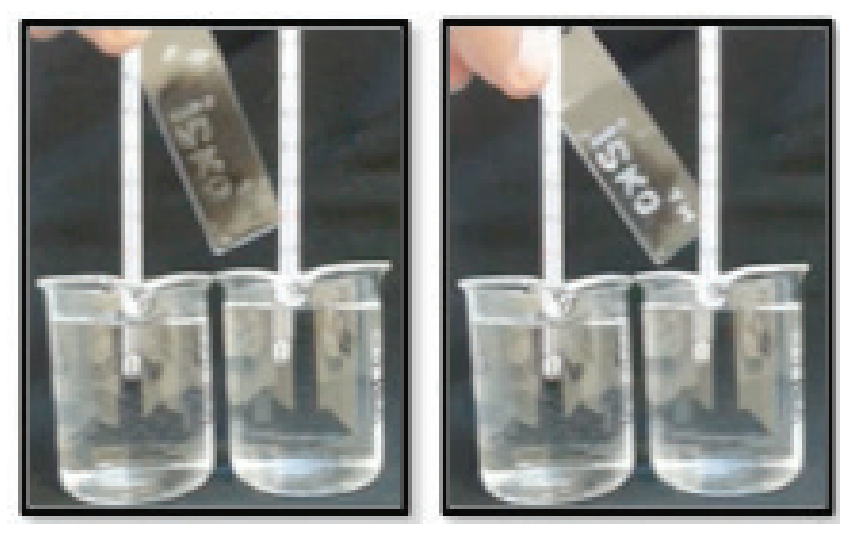

Figure 4. Thermal hydrophilic/hydrophobic transition of P(DMAEMA-co-HEMA) copolymer at different temperatures.

\section{CONCLUSIONS AND FUTURE WORK}

Prepared multifunctional polymers are found promising polyol constituents for polyurethane formulations in a wide range of temperature and $\mathrm{pH}$ without any phase separation or aggregation. Quaternized derivatives of those copolymers will be investigated as potential antimicrobial agents for textile industry. Copolymers having tertiary amine groups are improving not only indigo dyestuff uptake capability but also homogeneous indigo dyeability of the fabrics. Experiments on dye uptake as a function of quaternarization are ongoing. Well-defined copolymer structures having a tunable LCST are a great potential to achieve thermal stimuli adaptive textile surfaces.

\section{REFERENCES}

[1] Hu Jinlian, Adaptive and Functional Polymers, Textiles and Their Applications, Imperial College Press, 2011

[2] Wade A. Braunecker, Krzysztof Matyjaszewski, Controlled/living radical polymerization: Features, developments, and perspectives Progress in Polymer Science Volume 32, Issue 1, January 2007, p 93-146.

[3] Jaeger Werner, Bohrisch Joerg, Laschewsky Andre, Synthetic polymers with quaternary nitrogen atoms - Synthesis and structure of the most used type of cationic polyelectrolytes, Progress in Polymer Science 35, 511, 2010.

[4] Cheng Zhenping, Zhu Xinlin, Shi Z. L., Neoh K. G., Kang E. T., Polymer Microspheres with Permanent Antibacterial Surface from Surface-Initiated Atom Transfer Radical Polymerization, Ind. Eng. Chem. Res., 44, 7098, 2005. 